\title{
Unreadable Segment Recognition of Single-lead Dynamic Electrocardiogram Signals Based on Morphological Algorithm and Random Forest Classifier
}

\author{
Hanshuang Xie ${ }^{1}$, Huaiyu Zhu², Ji Zhao², Yisheng Zhao', Yun $\mathrm{Pan}^{2}$ \\ ${ }^{1}$ Research and Development Department, Hangzhou Proton Technology Co Ltd, Hangzhou, China \\ ${ }^{2}$ College of Information Science and Electronic Engineering, Zhejiang University, Hangzhou, China
}

\begin{abstract}
Recognizing unreadable electrocardiogram (ECG) signals could reduce the error rate of automatic software analysis and improve the interpretation efficiency of doctors, especially for single-lead dynamic ECGs. In this paper, we propose an unreadable ECG segment recognition method based on morphological algorithm and random forest classifier (RFC). The single-lead ECG signals are first filtered and normalized for morphological opening and closing operation, to generate detection sequences with more obvious $Q R S$ waves, since the large amplitudes introduced by motion interference could be suppressed during this procedure. Then features such as Shannon entropy and kurtosis are extracted and the RFC is used for unreadable segment classification. A total of 3354 readable segments and 2199 unreadable segments with a length of 4 seconds are obtained from 37 patients for method evaluation. The accuracy of our method (92.94 $\pm 0.93 \%)$ is significantly higher than that of the method without morphological algorithm (85.68 $\pm 1.30 \%)$. Moreover, we also used the " $N$ " and " $\sim$ " categories of the database from PhysioNet/CinC Challenge 2017 for further verification, and the accuracy of the proposed method $(93.75 \pm 0.69 \%)$ is significantly higher than that of the model without morphological processing $(82.25 \pm 1.06 \%)$ as well.
\end{abstract}

\section{Introduction}

The patch-type electrocardiogram (ECG) monitoring devices have enabled the recording of continuous dynamic ECG signals for more than 7 days. These ultralong-term ECGs could be applied to diagnose clinical diseases that cannot be confirmed using traditional 24hour Holter, e.g., unexplained syncope, occasional palpitation, paroxysmal painless myocardial ischemia, and paroxysmal angina pectoris [1]. However, electromagnetic interference, violent movement, and other noises in the users' environment can interfere with dynamic ECG signals and make them unreadable. The unreadable ECG segments would not help clinical diagnosis, since they would affect the judgment of the automatic analysis based software systems and cause a lot of trouble for the ECG interpretation of doctors. When the monitoring duration of ECGs increases to 7 days or even longer, a significant amount of unreadable ECG segments could affect the efficiency of doctors seriously. Many arrhythmias occur during daily activities, where the ECG signals are often disturbed by motion interference. If these ECG signals with motion interference are directly removed, some abnormal heartbeats could be missed in the examination. Therefore, in order to retain as many valid ECG signals as possible, we could consider ECG signals disturbed by the noise that does not affect the Rpeak recognition as readable signals; and other ECG signals that completely submerged by noise, i.e., the Rpeaks of the ECGs could hardly be extracted, could be determined as unreadable signals.

The recognition of unreadable ECG segments could be regarded as the ECG signal quality classification problem, where the signals are divided into readable ones and unreadable ones. Since PhysioNet/CinC Challenge 2011, ECG signal quality assessment has become a focused issue in the field of ECG signal research. Zhao et al. [2] proposed a signal quality index (SQI) evaluation mechanism of single-lead ECG signal based on simple heuristic fusion and fuzzy comprehensive evaluation, where the features such as R-peak detection match qSQI, QRS wave power spectrum distribution pSQI, kurtosis kSQI and baseline relative power basSQI were determined, and the fuzzy synthesis was used to perform the classification. Zhang et al. [3] proposed a hierarchical clustering method based on the agglomerative nesting algorithm for QRS wave detection and single-cycle ECG signal extraction according to three signal quality indexes of pSQI, kSQI and basSQI. The results showed that this algorithm is suitable for ECG quality assessment under different motion conditions. Zaunseder et al. [4] extracted simple spectral features and used the ensembles of decision trees to classify the ECG signals, yielding an accuracy of $90.4 \%$. Considering the chosen feature space, it is hardly feasible to further improve the accuracy. Li et 
al. [5] proposed an ECG signal quality classification algorithm with a total of 13 signal quality metrics derived from segments of ECG waveforms. A support vector machine (SVM) was trained to perform the classification and an accuracy of $88.07 \pm 0.32 \%$ was achieved in the 5fold cross-validation. Zhang et al. [6] proposed an ECG quality classification method based on kernel support vector machine (KSVM) and genetic algorithm (GA) to analyze and quantify the power spectrum, baseline drifts, amplitude difference, and other time-domain features to form the feature matrix. Finally, the feature matrix was assessed using KSVM and GA to determine the ECG quality results. This method got an accuracy of $91.8 \%$ on a database from PhysioNet/CinC Challenge 2011. Orphanidou et al. [7] proposed a method to calculate wavelet entropy using the heart rate variability signal. With the SVM, the sensitivity and specificity were $94 \%$ and $98 \%$ on data from the same sensor, and $91 \%$ and $97 \%$ on data from a different sensor. Although this method improves the classification accuracy, it requires an accurate acquisition of QRS.

In this paper, we propose an unreadable ECG segment recognition method based on morphological algorithm and random forest classifier (RFC). We evaluated our method based on the self-collected data and the "N" and " $"$ " categories of the database from PhysioNet/CinC Challenge 2017. The results showed that our method outperformed the method without morphological algorithm significantly, as $92.94 \pm 0.93 \%$ and $93.75 \pm$ $0.69 \%$ for the above-mentioned dataset, respectively.

\section{Method}

The proposed method contains the following steps: 1) signals are divided into segments of 4 seconds; 2) each segment is filtered by a finite impulse response (FIR) band-pass filter with a cut-off frequency range between $0.67 \mathrm{~Hz}$ and $40 \mathrm{~Hz} ; 3$ ) execute signal normalization and morphological opening and closing operation; 4) extract features and train the RFC model; 5) determine unreadable ECG segments for future ECG signals. The block diagram of our method is shown in Figure 1.

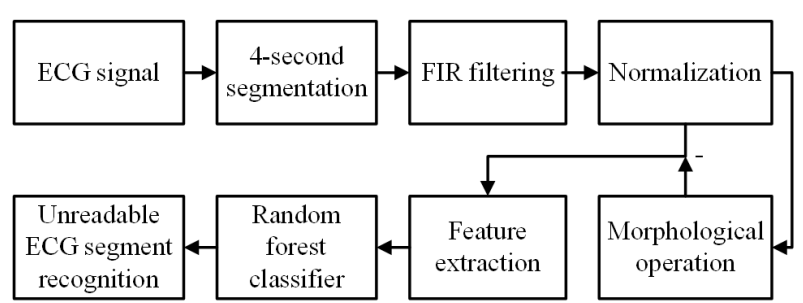

Figure 1. The block diagram of the proposed method.

\subsection{Preprocessing}

In this study, the FIR band-pass filter was used as the basic filter to remove the noise. The cut-off frequency of the filter was set between $0.67-40 \mathrm{~Hz}$ to better remove the baseline wandering, and retain most of the useful information of ECG signals.

Then, we applied the signal normalization to normalize the ECG signals to the range of $[-0.5,0.5]$. The processing formula is as the Equation (1):

$$
\operatorname{sigNor}(v a l)=\frac{v a l-\min (v a l)}{\max (v a l)-\min (v a l)}-0.5
$$

Finally, the morphological opening operation was applied on sigNor to obtain openSig, and then morphological closing operation was applied on openSig to get openCloseSig. The result of the difference between two sequences, i.e., sigNor - openCloseSig, is the signal pve required by the classification algorithm.

\subsection{Feature extraction}

By expanding the earlier published papers [2,8-11], eight signal quality indicators were extracted in our method from the signal pve, as shown in Table 1.

Table 1. Features extracted in the proposed method.

\begin{tabular}{ll}
\hline Feature name & Description \\
Kurtosis & $\begin{array}{l}\text { The measurement of the signal } \\
\text { symmetry }\end{array}$ \\
Skewness & $\begin{array}{l}\text { The measurement of the signal's } \\
\text { Gaussian property } \\
\text { The measurement of the time-serie } \\
\text { complexity }\end{array}$ \\
maxCorr & The maximum value of the \\
LZc & autocorrelation of ECG signals \\
meanECG & Lempel-Ziv complexity of ECG \\
stdECG & The mean value of ECG \\
validAmp & $\sqrt{\frac{\operatorname{sum}\left(E C G^{2}\right)}{\text { length(ECG) }}}$ \\
\hline
\end{tabular}

\subsection{Modeling}

In this study, RFC was used to perform classification. Each of the 500 decision trees was constructed with the Gini coefficient as a measurement. The classification ability of a single tree may be very small, but after a large number of decision trees are randomly generated, a test sample can select the most probable classification after counting the classification results of each tree.

\subsection{Dataset}

In this work, the patch-type single-lead ultra-long-term 
ECG recorder carePatch (NMPA\#ZJ20202070050) was used to collect clinical ECG signals of patients with a 256 $\mathrm{Hz}$ sampling rate, a 12-bit resolution and a dynamic range of $10 \mathrm{mV}$. The ECG signals of 37 clinical patients in Zhejiang Hospital were obtained and each ECG signal was divided into segments of 4 seconds. As no golden standard for the judgment of the signal quality has been proposed so far, in this paper, the only standard of data labelling rules of carePatch data is the QRS recognition accuracy. ECG segments with no more than 1 QRS recognition error were identified as readable segments, and segments with 2 or more recognition errors, whose Rpeaks position and QRS cannot be recognized, were regarded as unreadable segments. As a result, a total of 3354 readable segments and 2199 unreadable segments were labelled as the first dataset.

Furthermore, we divided all the noise data (labelled as " $")$ and part of the normal data (labelled as "N") in PhysioNet/CinC Challenge 2017 into segments of 4s, and normal segments from noise data were removed. In total, 1896 normal segments and 1519 noise segments were obtained as the second dataset.

\section{Results}

Both the datasets were randomly divided into the training set $(80 \%)$ and the testing set $(20 \%)$. For the first carePatch database, an average accuracy of $92.94 \pm 0.93 \%$ was observed using the five-fold cross-validation on the training set. Then the model was verified on the testing set. The receiver operating characteristic (ROC) curve is shown in Figure 2 with an area under the curve (AUC) of 0.97. Meanwhile, the results of the method without morphological processing before feature extraction were derived as well. The average accuracy of five-fold crossvalidation was $85.68 \pm 1.30 \%$. The ROC curve of the classification is shown in Figure 3 with an AUC of 0.91. The confusion matrix of both the procedure is shown in Table 2 .

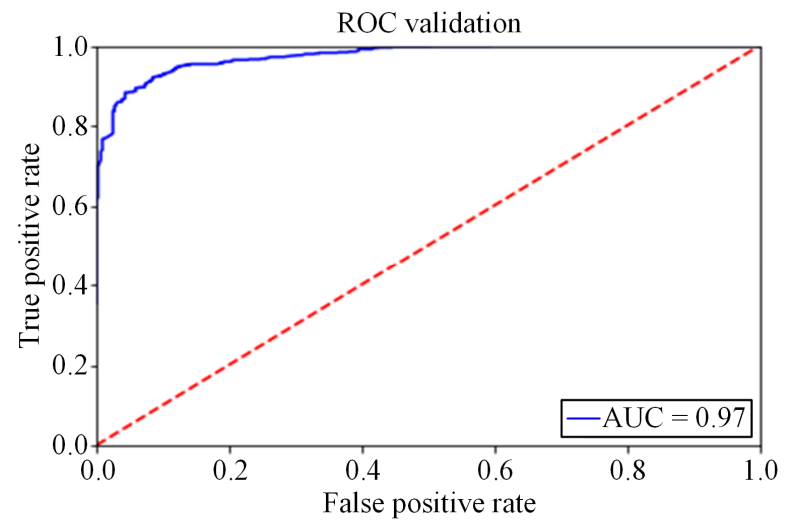

Figure 2. The ROC on carePatch dataset with the morphology processing.

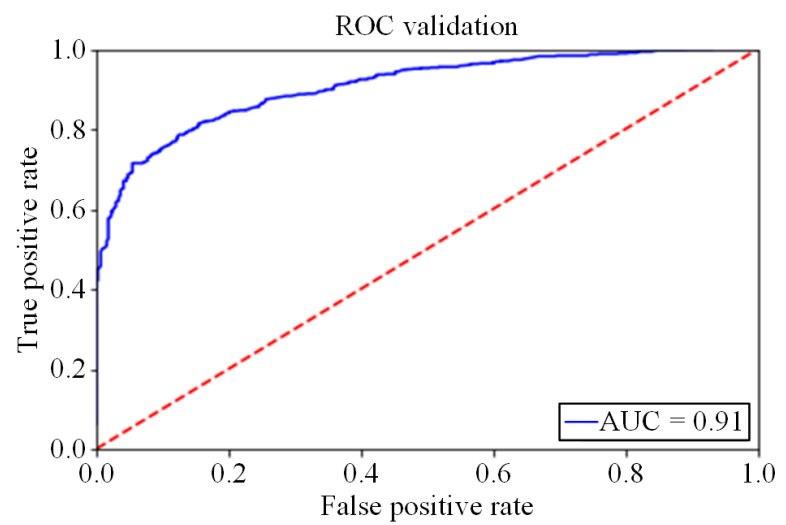

Figure 3. The ROC on carePatch dataset without the morphology processing.

Table 2. The confusion matrix on the carePatch dataset ("w/o" refers to without the morphology processing, and "w/" refers to with the morphology processing).

\begin{tabular}{|c|c|c|c|c|c|}
\hline \multirow{3}{*}{\multicolumn{2}{|c|}{$\begin{array}{c}0=\text { unreadable } \\
1=\text { readable }\end{array}$}} & \multicolumn{4}{|c|}{ Prediction label } \\
\hline & & \multicolumn{2}{|c|}{$\mathrm{w} / \mathrm{o}$} & \multicolumn{2}{|c|}{$\mathrm{w} /$} \\
\hline & & 0 & 1 & 0 & 1 \\
\hline True & 0 & 327 & 111 & 391 & 47 \\
\hline label & 1 & 91 & 582 & 48 & 625 \\
\hline
\end{tabular}

By this comparison, it is revealed that the classification accuracy of the method with the morphology processing is significantly higher than that without the morphology processing. In order to further evaluate the effectiveness of our method, we used the second PhysioNet/CinC Challenge 2017 dataset for verification. The classification accuracy of the method with morphology processing is significantly higher. After morphological processing, the average accuracy of five-fold cross-validation (93.75 \pm $0.69 \%)$ is also much higher than that without morphology processing $(82.25 \pm 1.06 \%)$. The ROCs are shown in Figures 4-5 and the confusion matrix is shown in Table 3.

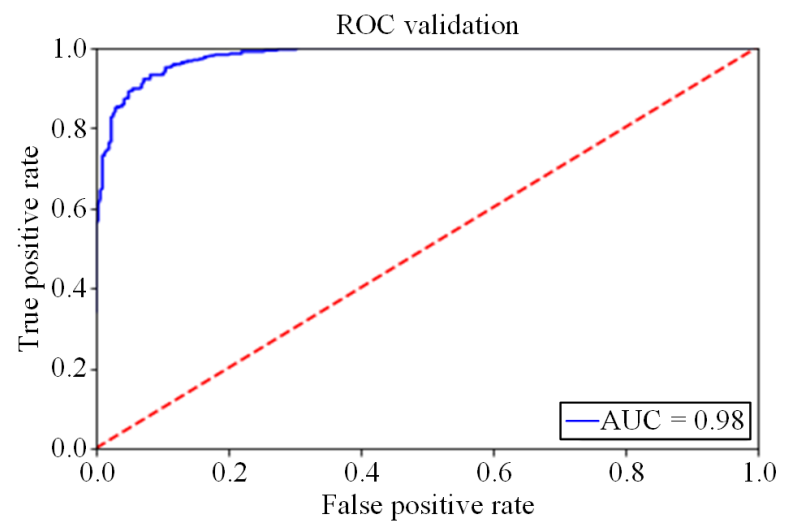

Figure 4. The ROC on PhysioNet/CinC Challenge 2017 dataset with the morphology processing. 


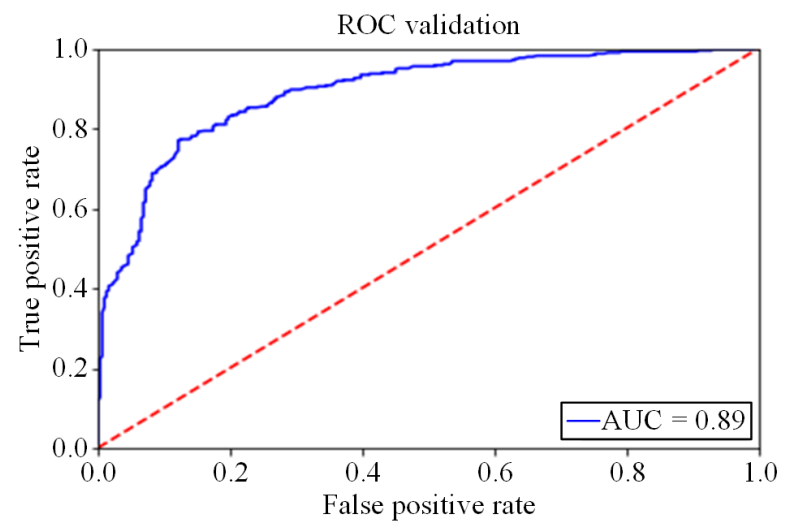

Figure 5. The ROC on PhysioNet/CinC Challenge 2017 dataset without the morphology processing.

Table 3. The confusion matrix on the PhysioNet/CinC Challenge 2017 dataset ("w/o" refers to without the morphology processing, and "w/" refers to with the morphology processing).

\begin{tabular}{cccccc}
\hline \hline \multirow{2}{*}{$0=$ unreadable } & \multicolumn{4}{c}{ Prediction label } \\
\cline { 2 - 6 } $1=$ readable & \multicolumn{3}{c}{ w/o } & \multicolumn{3}{c}{ w/ } \\
\cline { 2 - 6 } & 0 & 1 & 0 & 1 \\
\hline True & 0 & 225 & 79 & 274 & 30 \\
label & 1 & 47 & 331 & 22 & 356 \\
\hline \hline
\end{tabular}

The accuracy, precision, recall rate and F1 score of the two methods on the two datasets are shown in Table 4.

Table 4. Classification results ("CP" refers to carePatch dataset, "PC" refers to PhysioNet/CinC Challenge 2017 dataset, "w/o" refers to without the morphology processing, and " $\mathrm{w} /$ " refers to with the morphology processing).

\begin{tabular}{ccccc}
\hline $\begin{array}{c}\text { Data, } \\
\text { method }\end{array}$ & $\begin{array}{c}\text { Precision } \\
(\%)\end{array}$ & $\begin{array}{c}\text { Accuracy } \\
(\%)\end{array}$ & $\begin{array}{c}\text { Recall } \\
(\%)\end{array}$ & $\begin{array}{c}\text { F1 } \\
(\%)\end{array}$ \\
\hline CP, w/o & 81.8 & 78.2 & 74.7 & 76.4 \\
CP, w/ & 91.5 & 89.1 & 89.3 & 89.2 \\
PC, w/o & 81.5 & 82.7 & 74.0 & 78.1 \\
PC, w/ & 92.4 & 92.6 & 90.1 & 91.3 \\
\hline
\end{tabular}

\section{Conclusion}

In this paper, we present a method for recognizing unreadable segments of single-lead ECG signals based on morphological algorithm and random forest classifier. Experimental results showed that the morphological processing is essential to the recognition of unreadable single-lead ECG signals. In the future, we would try to adopt some new features to further improve the classification accuracy of the unreadable ECG segments from single-lead dynamic ECG signals.

\section{Acknowledgments}

We would like to thank the patients who enrolled in this research and the doctors of Zhejiang Hospital.

\section{References}

[1] X. Chen, M. Liu, and J. Yang. "Clinical application of single lead long-term ambulatory electrocardiography," Journal of Practical Electrocardiology, vol. 27, no. 4, pp. 269-272, Aug. 2018.

[2] Z. Zhao and Y. Zhang. "SQI quality evaluation mechanism of single-lead ECG signal based on simple heuristic fusion and fuzzy comprehensive evaluation," Frontiers in Physiology, vol. 9, pp. 727, Jun. 2018.

[3] Y. Zhang and Z. Zhao. "Evaluation of single-lead ECG signal quality with different states of motion," in Proc. IEEE 10th Int. Congr. Image Signal Process., BioMed. Eng. Inf. (CISP-BMEI), 2017, pp. 1-7.

[4] S. Zaunseder, R. Huhle, and H. Malberg. "CinC challenge-Assessing the usability of ECG by ensemble decision trees," In Proc. 2011 Computing in Cardiology, vol. 38, 2011, pp. 277-280.

[5] Q. Li, C. Rajagopalan, and G. D. Clifford. "A machine learning approach to multi-level ECG signal quality classification," Computer Methods and Programs in Biomedicine, vol. 117, no. 3, pp. 435-447, Dec. 2014.

[6] Y. Zhang, C. Liu, S. Wei, C. Wei, and F. Liu. "ECG quality assessment based on a kernel support vector machine and genetic algorithm with a feature matrix," Journal of Zhejiang University SCIENCE C, vol. 15, no. 7, pp. 564-573, Jul. 2014.

[7] C. Orphanidou and I. Drobnjak. "Quality assessment of ambulatory ECG using wavelet entropy of the HRV signal," IEEE Journal of Biomedical and Health Informatics, vol. 21, no. 5, pp. 1216-1223, Sep. 2017.

[8] S. Liu, J. Lu, L. Hao, and G. Hu. "Detection of QRS complex using mathematical morphology and wavelet transform," J. Tsinghua Univ. (Sci. \& Tech.), vol. 44, no. 6, pp. 852-855, Jun. 2004.

[9] Y. Zhang, S. Wei, L. Zhang, and C. Liu. "Comparing the performance of random forest, SVM and their variants for ECG quality assessment combined with nonlinear features," Journal of Medical and Biological Engineering, vol. 39, no. 3, pp. 381-392, Apr. 2018.

[10] Y. Xu, M. Luo, and T. Li. "Study on ECG signal denoising algorithm based on CEEMDAN and wavelet threshold," Modern Electronics Technique, vol. 41, no. 7, pp. 45-48, Apr. 2018.

[11] Y. Zhang. "Research of quality assessment of ECGs collected via mobile device," Ph.D. dissertation, School of Control Science and Engineering, Shandong University, Jinan, China, 2015.

Address for correspondence:

Yun Pan

Room 504, Laoshengyi Building, Yuquan Campus, Zhejiang University, No.38 Zheda Road, Hangzhou, Zhejiang, China panyun@zju.edu.cn 